\title{
Alterações Eletroneurofisiológicas em Anestesia com Sevoflurano: Estudo Comparativo entre Pacientes Saudáveis e Pacientes com Paralisia Cerebral *
}

\section{Electroneourophysiological Changes in Anesthesia with Sevoflurane: Comparative Study between Healthy and Cerebral Palsy Patients}

Sérgio Silva de Mello, $T_{S A}{ }^{1}$, Renato Ângelo Saraiva, TSA $^{2}$

\begin{abstract}
RESUMO
Mello SS, Saraiva RA - Alterações Eletroneurofisiológicas em Anestesia com Sevoflurano: Estudo Comparativo entre Pacientes Saudáveis e Pacientes com Paralisia Cerebral
\end{abstract}

\begin{abstract}
Justificativa e Objetivos - Estudos avaliando a farmacodinâmica de drogas anestésicas em Paralisia Cerebral $(P C)$ são escassos. O objetivo deste estudo foi comparar a resposta eletroneurofisiológica de pacientes normais e com PC à anestesia com sevoflurano, utilizando o EEG bispectral (BIS) e potencial evocado somatossensitivo de curta latência (PESS) como técnica de monitorização do grau de profundidade anestésica.
\end{abstract}

Método - Foram selecionados 24 pacientes de 3 a 18 anos, candidatos a cirurgias na Rede Sarah de Hospitais do Aparelho Locomotor, divididos em 2 grupos: 1. PC - 12 crianças com PC tipo espástica; 2. Controle (C) - 12 crianças sem doença do SNC. A anestesia foi realizada com sevoflurano associado a $\mathrm{N}_{2} \mathrm{O}$ a $60 \%$ em ventilação assistida. As variáveis BIS e ondas N9, N13, N19 e P/N 22 do PESS - latência e amplitude - foram avaliadas nas frações expiradas de sevoflurano (FEsev) de 1,2 e $2,5 \%$ (0,5 e 1 CAM). Foram monitorizadas a temperatura e a $P_{E T} C_{2}$. Para análise foram utilizadas média e desvio-padrão para o BIS, e média da variação percentual dos valores de latência e amplitude das ondas do PESS, nas duas concentrações do anestésico.

Resultados - Não houve diferença de sexo, idade, peso e temperatura entre os grupos. Sob efeito da anestesia, os valores de BIS foram mais reduzidos no grupo PC, sendo a diferença entre os grupos estatisticamente significante com a FEsev 2,5\% (30,3 x 37,5; $p<0,05)$. O aumento percentual da latência das ondas do PESS foi maior no grupo PC.

Conclusões - Os resultados sugerem um efeito depressor maior do anestésico no grupo com PC, sendo esta diferença captada pelo BIS e PESS.

UNITERMOS: ANESTÉSICOS, Volátil: sevoflurano; DOENÇA, Neurológica: paralisia cerebral; MONITORIZAÇÃO: índice bispectral, potencial evocado somatossensitivo

\footnotetext{
* Recebido da (Received from) Rede Sarah de Hospitais do Aparelho Locomotor, Brasilia, DF

1. Anestesiologista da Rede Sarah de Hospitais do Aparelho Locomotor

2. Coordenador de Anestesiologia da Rede Sarah de Hospitais do ApareIho Locomotor
}

Apresentado (Submitted) em 11 de junho de 2002

Aceito (Accepted) para publicação em 27 de agosto de 2002

Correspondência para (Mail to)

Dr. Sérgio Silva de Mello

Av. Amazonas, 5953 - Gamaleira

30510-000 Belo Horizonte, MG

E-mail: sergiomello@bhz.sarah.br

(C) Sociedade Brasileira de Anestesiologia, 2003

\author{
SUMMARY \\ Mello SS, Saraiva RA - Electroneourophysiological Changes in \\ Anesthesia with Sevoflurane: Comparative Study between He- \\ althy and Cerebral Palsy Patients
}

Background and Objectives - There are very few studies on anesthetic drugs pharmacodynamics in patients with Cerebral Palsy (CP). This study aimed at comparing electroneurophysiological responses in healthy and CP patients, using bispectral index (BIS) and short-latency somatosensory evoked potential (SEP) to monitore sevoflurane-induced central nervous system (CNS) electroneurophysiological changes.

Methods - Twenty four patients aged 3 to 18 years, scheduled for surgical procedures were allocated in two groups: 1. CP - 12 patients with spastic CP; 2. Control (C) - 12 patients with no neurological disease. Anesthesia was induced with sevoflurane and $60 \% \mathrm{~N}_{2} \mathrm{O}$ in assisted ventilation. BIS and $\mathrm{N} 9$, N13, N19 and N/P22 SEP waveforms - amplitude and latency were recorded at baseline and at $1.2 \%$ and $2.5 \%$ end-tidal sevoflurane concentration (ETsev), corresponding to 0.5 and 1 MAC, respectively. Monitoring consisted of temperature, ETsev and $P_{E T} \mathrm{CO}_{2}$. For statistical analysis, BIS mean and standard deviation as well as means percentage variation of SEP waveforms latency and amplitude in both anesthetic concentrations, were used.

Results - There were no significant differences in gender, age, weight and temperature between groups. Under anesthesia, $B I S$ values were lower in the CP group, whit statistical significance only at $2.5 \%$ ETsev $(30.3 \times 37.5 ; p<0.05)$. Percentage SEP waveforms latency increase was higher in the CP group.

Conclusions - Results suggest a deeper anesthetic depressing effect in the CP group and this difference was captured by BIS and SEP waves.

KEY WORDS: ANESTHETICS, Volatile: sevoflurane; DISEASE, Neurologic: cerebral palsy; MONITORING: bispectral index, somatosensory evoked potential

\section{INTRODUÇÃO}

paralisia cerebral (PC) é uma desordem do movimento Aprovocada por uma lesão não progressiva no cérebro em desenvolvimento ${ }^{1}$. Sua incidência vem se mantendo constante nos últimos anos, apesar dos avanços da Medicina moderna. Uma grande parcela dos pacientes com PC irá se submeter a um procedimento anestésico em algum momento de suas vidas, seja para fins propedêuticos ou terapêuticos.

O perfil farmacodinâmico das drogas anestésicas nesse grupo de pacientes é pouco estudado. Frei e col. realizaram um estudo no qual demonstraram que crianças com PC seriam mais sensíveis à ação do halotano que crianças saudáveis ${ }^{2}$. 
O índice bispectral (BIS) e o potencial evocado somatossensitivo de curta latência (PESS) registram as alterações eletroneurofisiológicas provocadas pelos anestésicos sobre o sistema nervoso central (SNC), cérebro e estruturas subcorticais, respectivamente, variando seus parâmetros de maneira dose-dependente com estas drogas ${ }^{3,4}$. Seria interessante observar o comportamento desses monitores em pacientes portadores de lesão cerebral.

Este estudo visou comparar as alterações eletroneurofisiológicas provocadas pelo sevoflurano sobre o SNC de crianças normais e com PC, utilizando o BIS e o PESS como monitores capazes de aferir e quantificar estas alterações. Além disso, observou-se o comportamento do BIS nas crianças com PC, a fim de se verificar a possibilidade da sua utilização nesse grupo de pacientes.

\section{MÉTODO}

Após aprovação do Comitê de Ética da Rede Sarah de Hospitais do Aparelho Locomotor e obtenção do consentimento pós-informado dos responsáveis pelos componentes da amostra, 24 pacientes de ambos os sexos, com idades entre 3 e 18 anos, foram divididos em 2 grupos da seguinte forma: 1. PC - composto de 12 pacientes com PC tipo espástica, diagnosticada de acordo com critérios clínicos ${ }^{5} ; 2$. Controle 12 pacientes sem doença do SNC, estado físico ASAI. Os critérios de exclusão foram doenças sistêmicas, uso de benzodiazepínicos ou baclofen, contra-indicação à anestesia inalatória, hipertermia e obesidade.

Os pacientes não receberam medicação pré-anestésica. A indução e a manutenção da anestesia foram com sevoflurano associado a $\mathrm{N}_{2} \mathrm{O}$, com fluxo de $3 \mathrm{~L}$. $\mathrm{min}^{-1}$, circuito valvulare com absorvedor de $\mathrm{CO}_{2}$. Todos os pacientes foram submetidos à intubação orotraqueal. Em todos os casos associou-se anestesia regional, peridural simples ou bloqueio de plexo braquial, com ropivacaína ou bupivacaína nas doses habituais $\left(\leq 2 \mathrm{mg} \cdot \mathrm{kg}^{-1}\right)$. Foram monitorizados continuamente fração expirada final de $\mathrm{CO}_{2}\left(\mathrm{P}_{\mathrm{ET}} \mathrm{CO}_{2}\right)$ e sevoflurano, temperatura nasofaríngea em graus Celsius, oximetria de pulso e pressão arterial não-invasiva em $\mathrm{mmHg}$. A ventilação foi assistida, no intuito de se manter $\mathrm{P}_{\mathrm{ET}} \mathrm{CO}_{2}$ entre 30 e $50 \mathrm{mmHg}$. Foram avaliados os efeitos do sevoflurano sobre o BIS e as ondas do PESS no pré-anestésico imediato, quando possível, e nas concentrações de $1,2 \%$ e $2,5 \%$, administrados de forma crescente, sendo a fração expirada mantida durante 15 minutos antes das aferições. As aferições foram realizadas antes do início da cirurgia na maior parte dos pacientes. Naqueles em que foi realizado após a incisão, o estímulo cirúrgico não alterou a aferição, já que foi associada anestesia regional. Foram considerados valores basais, equivalente ao paciente acordado, quando este apresentava reações características do despertar, o BIS apresentava-se acima de 95, as ondas do PESS sem depressão e a concentração alveolar do anestésico de 0 (zero).

O índice bispectral foi registrado a partir do aparelho A-1000 (Aspect Medical System, Natick, MA), sendo utilizada mon- tagem referencial. Os eletrodos foram do tipo Zipprep (Aspect Medical System, Natick, MA), sendo fixados nas posições Fp1, Fpz e Fp2 (sistema internacional 10-20). Aimpedância foi mantida abaixo de $2 \Omega$ e a banda de freqüência situada entre 05 e $30 \mathrm{~Hz}$. O índice bispectral de 0 a 100 e as ondas dos dois canais do BIS foram registrados a partir de épocas sucessivas de 2 segundos de duração e atualizados a cada 5 segundos. O valor considerado para o estudo foi a média dos valores encontrados nos 45 segundos que antecederam o momento estipulado para aferição. Após sua obtenção, os dados foram registrados em formulário próprio, conforme especificado no desenho do estudo.

O PESS do nervo mediano foi registrado utilizando-se o aparelho Dantek - Keypoint versão 1.6 (Denmark), sendo os eletrodos de superfície fixados nas seguintes posições: Ponto de Erb's, Cll e C3' ou C4 (Sistema internacional 10-20). Aimpedância dos eletrodos foi mantida inferior a $5 \Omega$, sendo $F z$ empregado como referência. A intensidade do estímulo foi ajustada até se atingir o limiar de resposta motora (Thumb Twitch). Para cada PESS, 300 a 500 impulsos de corrente constante foram repetidos com duração de 0,2 ms e razão de descarga de $5 \mathrm{~Hz}$. A banda de filtragem foi ajustada entre 20 $\mathrm{Hz}$ (baixa) e $3000 \mathrm{~Hz}$ (alta). O sinal foi amplificado, permitindo uma sensibilidade de $5 \mu$ v por divisão, sendo a varredura de $5 \mathrm{~ms}$ por divisão. As médias foram realizadas em duplicata. Foram aferidos latência, em milissegundos (ms), e amplitude, em microvolts ( $\mu \mathrm{v})$, das seguintes ondas: N9, N13, N19 e P/N22, nas concentrações expiradas do anestésico especificadas no desenho do estudo. Esses registros foram realizados cerca de 1 minuto após o registro do BIS, sendo os resultados impressos em papel e gravados em disco magnético do próprio aparelho, e posteriormente analisados por um neurofisiologista que desconhecia o quadro do paciente. A análise estatística foi realizada utilizando-se o programa SPSS. Foram empregados valores da média e desvio padrão das variáveis consideradas para análise, exceto as ondas do PESS. Como os valores absolutos destas ondas podem variar com a idade, os valores de latência e amplitude considerados para análise foram os referentes à variação percentual média dessas variáveis, após a concentração alveolar do anestésico ser aumentada para 2,5\% e mantida por 15 minutos, ou seja, qual o valor percentual médio da diminuição da amplitude e do aumento da latência, quando a concentração expirada final do anestésico foi aumentada de $1,2 \%$ para $2,5 \%$. Utilizou-se o teste $t$ de Student simples para comparação entre os grupos e o teste de medidas múltiplas para as comparações dentro de cada grupo, nas diferentes concentrações do sevoflurano. Análise de regressão múltipla e ANOVA também foram empregadas quando necessário.

\section{RESULTADOS}

Não houve diferença entre os grupos no que se refere a idade, sexo, peso e variação da temperatura corporal, sendo esta mantida em todos os casos acima de $35,3^{\circ}$ (Tabela I). 
Tabela I - Distribuição dos Pacientes nos Dois grupos - Paralisia Cerebral e Controle

\begin{tabular}{lcc}
\hline & Grupo PC & Grupo Controle \\
\hline Sexo M/F & $7 / 5$ & $4 / 8$ \\
Peso $(\mathrm{kg}){ }^{*}$ & $29,95 \pm 14,46$ & $40,91 \pm 15,95$ \\
Idade (anos) ${ }^{*}$ & $9,67 \pm 4,21$ & $12,08 \pm 3,6$ \\
\hline
\end{tabular}

*Valores expressos pela Média \pm DP

O grupo PC foi composto de pacientes diplégicos (5), tetraplégicos (4) e hemiplégicos (3). Desses, 4 utilizavam anticonvulsivantes (fenobarbital, ácido valpróico e carbamazepina).

Os valores do índice bispectral nas diferentes concentrações propostas no estudo podem ser vistos na tabela II. Houve decréscimo nos valores médios dentro de cada grupo, à medida que se aumentava a concentração alveolar do anestésico.

Tabela II - Comparação entre os Valores de Média e Desvio Padrão do Índice Bispectral (BIS) nas Diferentes Concentrações de Anestésico, nos Grupos Paralisia Cerebral e Controle

\begin{tabular}{lcc}
\hline Concentração Sevoflurano & BIS - Paralisia Cerebral & BIS - Controle \\
\hline $0 \%$ & $97,75(0,45)$ & $97,58(0,67)$ \\
$1,2 \%$ & $55(13,07)$ & $60,17(9,21)$ \\
$2,5 \%$ & $30,33(7,06)$ & $37,50(9,41)^{\star}$ \\
\hline
\end{tabular}

${ }^{*} p<0,05$ - significativo

Na comparação entre os grupos, os valores de média do BIS no grupo PC foram inferiores ao grupo controle, sendo a dife- rença estatisticamente significante na concentração de $2,5 \%$ de sevoflurano $(p<0,05)$. Na concentração de $1,2 \%$, a média dos valores de BIS também foi inferior no grupo PC, embora sem significância estatística.

A realização do PESS basal não foi possível em alguns dos componentes da amostra, motivo pelo qual optamos por analisar os resultados obtidos durante as duas concentrações de sevoflurano. As ondas do PESS comportaram-se como esperado, com aumento da latência e diminuição da amplitude ao aumentar-se a concentração do anestésico, em ambos os grupos (Figuras 1 e 2).

A variação percentual dos valores da amplitude foram semeIhantes em ambos os grupos. No que se refere à latência das ondas do PESS, aquelas originadas de derivações tálamo-corticais tiveram um aumento estatisticamente significativo em ambos os grupos, quando do aumento da concentração do anestésico (Tabelas III e IV). Pode se observar ainda uma tendência a aumentos percentuais maiores desses valores no grupo PC, apesar de não existir diferença estatisticamente significante.

Tabela III - Comparação das Variações Percentuais $(\Delta \%)$ nos Valores Médios de Amplitude das Diferentes Ondas do PESS entre os Grupos Paralisia Cerebral e Controle, quando do Aumento da Concentração Alveolar Final de Sevoflurano de 1,2\% para 2,5\%

\begin{tabular}{lcc}
\hline Ondas PESS & $\Delta \%$ Grupo Paralisia Cerebral & $\Delta \%$ Grupo Controle \\
\hline N9 & $-10 \%$ & $-7,9 \%$ \\
N13 & $-3,5 \%$ & $-8,8 \%$ \\
N19 & $-50,7 \%$ & $-42 \%$ \\
P/N22 & $-40,7 \%$ & $-40,7 \%$ \\
\hline
\end{tabular}
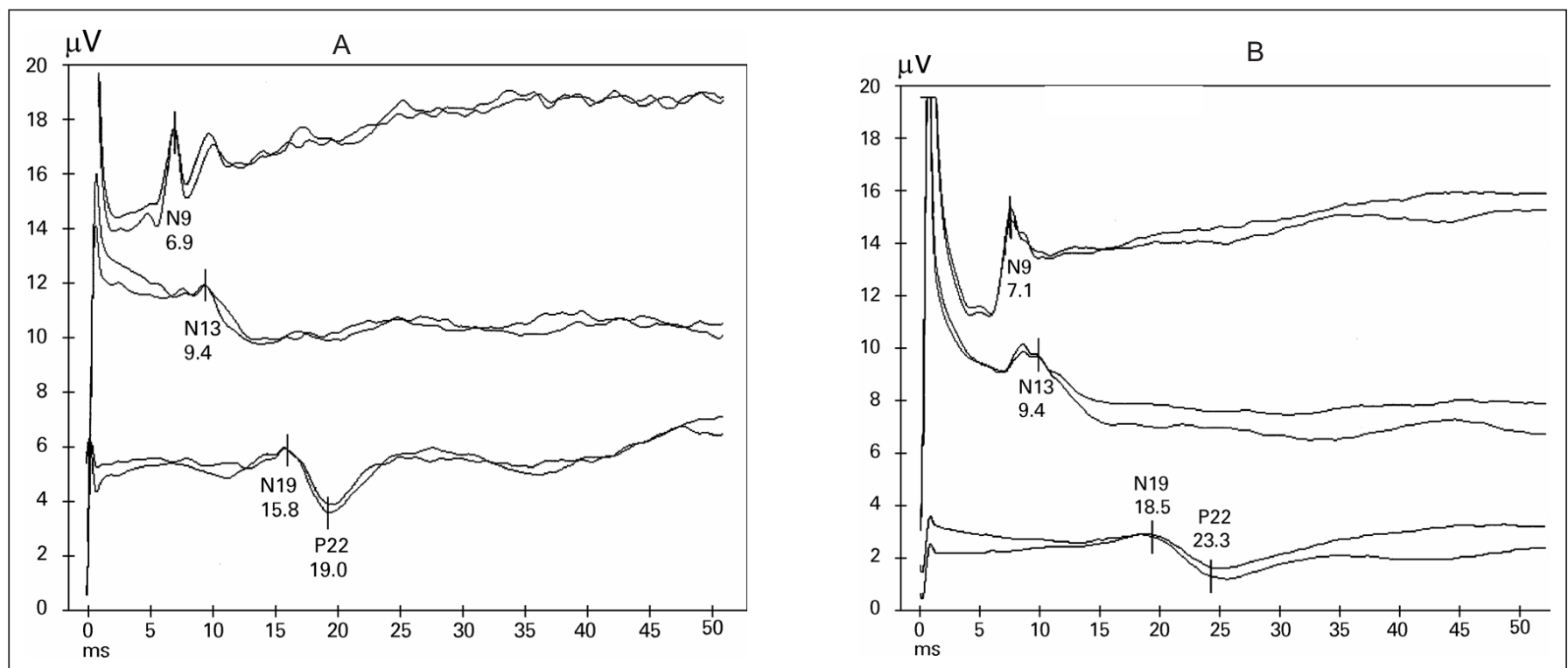

Figura 1 - Traçado de PESS de Paciente do Grupo Controle quando Acordado (A) e Anestesiado com Sevoflurano na Fração Expirada Final de $2,5 \%$, (B) Respectivamente.

Observa-se o aumento da latência (número abaixo da denominação da onda. Ex: N9-6,9) e a diminuição da amplitude, quando o paciente se encontra anestesiado, sobretudo nas derivações tálamo-corticais (N19 e P/N22) 


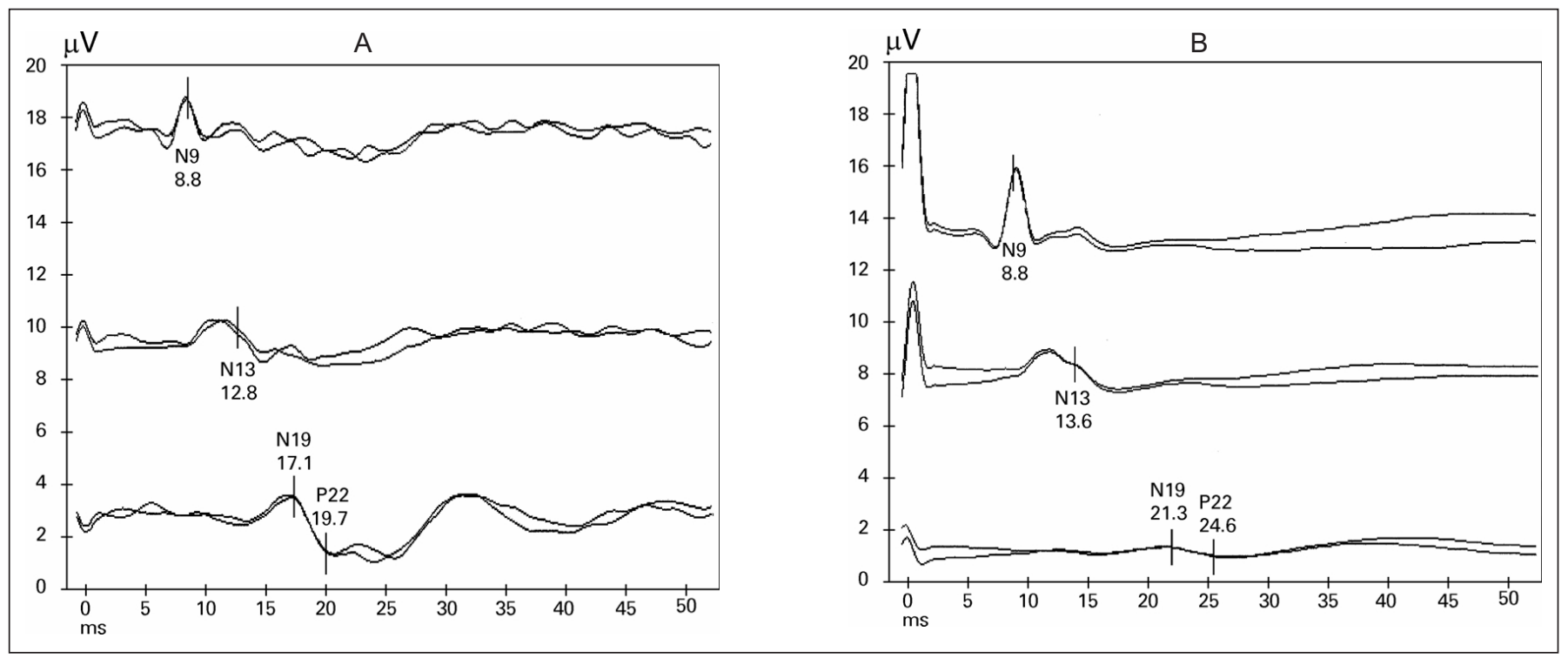

Figura 2 - Traçado de PESS de Paciente do Grupo Paralisia Cerebral quando Acordado (A) e Anestesiado com Sevoflurano na Fração Expirada Final de $2,5 \%$, (B) Respectivamente.

Observa-se o aumento da latência (número abaixo da denominação da onda. Ex: N9-6,9) e a diminuição da amplitude, quando o paciente se encontra anestesiado, sobretudo nas derivações tálamo-corticais (N19 e P/N22). Estas alterações são mais importantes neste grupo em relação ao grupo controle

Tabela IV - Comparação das Variações Percentuais $(\Delta \%)$ nos Valores Médios de Latência das Diferentes Ondas do PESS entre os Grupos Paralisa Cerebral e Controle, quando do Aumento da Concentração Alveolar Final de Sevoflurano de $1,2 \%$ para $2,5 \%$

\begin{tabular}{lcc}
\hline Ondas PESS & $\Delta \%$ Grupo Paralisia Cerebral & $\Delta \%$ Grupo Controle \\
\hline N9 & $4,0 \%$ & $0,1 \%$ \\
P/N13 & $3,1 \%$ & $2,8 \%$ \\
N19 & $13,2 \%$ & $9,7 \%$ \\
P/N22 & $11,9 \%$ & $10,5 \%$ \\
\hline
\end{tabular}

\section{DISCUSSÃO}

Em nosso estudo ficou demonstrado que, na população estudada, os pacientes com PC apresentaram alterações eletroneurofisiológicas mais pronunciadas que aqueles considerados saudáveis, quando submetidos às mesmas concentrações expiradas de sevoflurano. O índice bispectral decresceu de forma mais acentuada no grupo com PC, sugerindo uma sensibilidade maior desses pacientes à ação hipnótica do anestésico.

Os estudos acerca da farmacodinâmica das drogas anestésicas em pacientes com PC são escassos. Das drogas utilizadas em anestesia, os bloqueadores neuromusculares provavelmente foram aquelas que tiveram o maior número de estudos publicados ${ }^{6,7}$. Também drogas empregadas freqüentemente em PC, como baclofen e os anticonvulsivantes benzodiazepínicos, tiveram a sua interação com anestésicos inalatórios estudados ${ }^{8}$. Outros anticonvulsivantes, como nos casos da carbamazepina e do ácido valpróico, ou

Revista Brasileira de Anestesiologia

Vol. 53, № 2, Março - Abril, 2003 não demonstraram efeitos importantes sobre a ação dos anestésicos inalatórios com seu uso crônico, ou não têm demonstrada sua interação com os agentes inalatórios ${ }^{9}$. Nolam publicou recentemente um artigo de revisão acerca de anestesia em PC, chamando a atenção para as peculiaridades envolvendo o manuseio anestésico desses pacientes, porém sem se ater às características farmacodinâmicas e farmacocinéticas dos anestésicos nesse grupo específico de indivíduos ${ }^{8}$.

Frei e col. ${ }^{2}$, em 1997, estudaram crianças com PC e retardo mental grave no que se refere à sensibilidade ao halotano, utilizando a CAM como medida de potência da ação deste anestésico. Porém, para a obtenção da CAM seria necessária uma resposta motora adequada. A utilização desse instrumento de aferição em PC, em que a manifestação clínica principal é a desordem do movimento, poderia ocasionar um importante viés.

No presente estudo, procuramos empregar técnicas capazes de registrar e quantificar as alterações eletrofisiológicas provocadas pelo sevoflurano, e que não dependessem de nenhuma resposta subjetiva ou que pudesse ser modificada pela condição clínica dos pacientes estudados. Optamos, então, por utilizar o BIS e o PESS, que registram a atividade elétrica no córtex e estruturas subcorticais, respectivamente, e têm seus parâmetros comprovadamente alterados pelos agentes inalatórios de maneira dose-dependente, possibilitando assim um registro mais amplo das modificações ocorridas em todo SNC em função da ação do anestésico ${ }^{10,11}$.

Não foram encontrados estudos publicados nos quais utilizou-se o BIS em pacientes com PC. No nosso caso, este índice mostrou-se adequado para ser empregado como monitor neurofisiológico nesses indivíduos. Os valores basais foram quase idênticos em ambos os grupos, e houve um decrésci- 
mo linear à medida que se aumentou a concentração expirada do anestésico tanto nos pacientes saudáveis quanto naqueles com doença neurológica. Em 2000, Denman e col. consideraram adequada a utilização do BIS em crianças menores de 2 anos, baseando-se no comportamento do índice bispectral nesses pacientes, que seria semelhante ao das crianças maiores e adultos ${ }^{12}$. A partir do presente estudo, e empregando-se o mesmo raciocínio, podemos sugerir que o BIS poderia ser utilizado em crianças com PC, independentemente do tipo clínico, já que na amostra estudada havia pacientes portadores de diferentes formas clínicas de PC.

Quando anestesiados, os pacientes dos dois grupos demonstraram comportamento diferente do BIS em termos de sensibilidade ao anestésico, com valores de média menores no grupo PC em relação ao grupo controle. Esta diferença foi encontrada nas duas concentrações de sevoflurano, sendo estatisticamente significante a 1 CAM. Algumas hipóteses podem justificar tais achados.

Woodforth e col., em 1999, publicaram um estudo no qual foram encontradas evidências de que os anestésicos gerais com características hipnóticas, como o sevoflurano, agiriam suprimindo a atividade interneuronal no córtex cerebral, sendo este o mecanismo responsável pela inconsciência provocada por essas drogas ${ }^{13}$. Estudos em animais sugeriram ainda que este efeito ocorreria principalmente sobre os interneurônios corticais das lâminas III e $\mathrm{V}^{14}$.

Miller, em 1998, aventou a hipótese de que as dificuldades de aprendizado apresentadas pelas crianças com $\mathrm{PC}$ poderiam ser devidas a um prejuízo no funcionamento das fibras de associação corticais ${ }^{15}$. Além disso, Volpe sugeriu que lesões nos neurônios subcapsulares afetariam a organização cortical e a conectividade neuronal ${ }^{16}$.

Se confrontarmos as informações provenientes destes estudos, podemos formular a hipótese de que crianças com PC já estariam com as suas conexões interneuronais corticais prejudicadas. Conseqüentemente, a quantidade de anestésico necessário para bloquear o funcionamento destas células, previamente lesadas e com um número provavelmente menor de interconexões, seria menor nesses pacientes que naqueles em que as associações entre neurônios está preservada. Esta hipótese é convidativa, porém permanece apenas no campo especulativo.

A lesão em local mais específico do SNC, onde também atuassem os anestésicos gerais, poderia diminuir a necessidade dessas drogas nesses pacientes. Newman chamou atenção para a importância do tálamo e de suas conexões com o córtex para o mecanismo da atenção e consciência ${ }^{17}$. Angel também destacou o tálamo como um provável local de ação dos anestésicos no SNC ${ }^{14}$. Em alguns tipos clínicos de PC, como a tetraplegia espástica e as formas coreoatetósicas, pode existir lesão ou disfunção talâmica ${ }^{15}$. Alterações no funcionamento desta estrutura poderiam justificar uma necessidade menor de anestésico para bloqueio das funções celulares e obtenção do estado anestésico de inconsciência, registrado pelo BIS.

Os dados referentes ao PESS confirmaram os achados de outros autores ${ }^{11}$. Com o aumento da concentração alveolar do anestésico, os valores de amplitude dos pacientes diminuíram de forma considerável em ambos os grupos, como esperado, e houve um aumento na latência das ondas, sobretudo naquelas referentes aos geradores tálamo-corticais (N19/P22). No presente estudo, a latência de todas as ondas do grupo com PC apresentaram um aumento maior, em termos percentuais, em relação às crianças sem doença do SNC, porém sem significância estatística.

Existem limitações no presente estudo. Pode ter ocorrido um viés de prevalência, já que apenas os casos cirúrgicos de PC foram incluídos na amostra. Porém, graus variados de gravidade e diversos tipos clínicos da doença fizeram parte do estudo, permitindo a avaliação da resposta em diferentes graus de acometimento da doença. Além disso, O BIS ainda é um aparelho em estudo e aperfeiçoamento, possuindo limitações para uso rotineiro em Anestesiologia ${ }^{18,19}$. A utilização de anticonvulsivantes por alguns pacientes do grupo PC poderia ter modificado algum parâmetro eletrofisiológico. Porém, aqueles que faziam uso de drogas que sabidamente reduziam a CAM, como benzodiazepínicos, foram excluídos do estudo.

Pacientes com PC mostraram-se mais sensíveis à ação do sevoflurano sobre o SNC que aqueles considerados saudáveis, quando utilizados o BIS e o PESS como técnicas de monitorização das alterações eletroneurofisiológicas provocadas pelo anestésico. Estes achados podem ser devido a uma diminuição das interconexões neuronais nesses pacientes. Futuros estudos devem ser realizados com objetivo de esclarecer essa hipótese, pois não apenas poderiam responder a esta pergunta, como também a outros importantes questionamentos, como aqueles relacionados ao local e mecanismo de ação das drogas anestésicas inalatórias.

\section{Electroneourophysiological Changes in Anesthesia with Sevoflurane: Comparative Study between Healthy and Cerebral Palsy Patients}

Sérgio Silva de Mello, TSA, M.D., Renato Ângelo Saraiva, TSA, M.D.

\section{INTRODUCTION}

Cerebral palsy (CP) is a movement disorder caused by a non progressive injury in the developing brain ${ }^{1}$. Its incidence is remaining constant in recent years, is spite of modern medicine advances. A large number of CP patients will eventually be submitted to an anesthetic procedure, both for propedeutic and therapeutic purposes.

The pharmacodynamic profile of anesthetic drugs in this group of patients is not well studied. Frei et al. have shown that CP children are more sensitive to halothane as compared to healthy children ${ }^{2}$.

Revista Brasileira de Anestesiologia Vol. 53, № 2, Março - Abril, 2003 
Bispectral index and short-latency somatosensory evoked potential (SEP) record electroneurophysiological changes induced by anesthetics in central nervous system (CNS) brain and subcortical structures. Parameters vary in a dose-dependent manner ${ }^{3,4}$. It would be interesting to observe readings of such monitors in CP patients.

This study aimed at comparing sevoflurane-induced electroneurophysiological changes in the CNS of healthy and CP children, using BIS and SEP to check and quantify such changes. In addition, BIS behavior in CP children was observed to assess the use of BIS in this group of children.

\section{METHODS}

After "Rede Sarah de Hospitais do Aparelho Locomotor" Ethical Committee approval and the informed consent of parents or tutors, 24 patients of both genders, aged 3 to 18 years, were divided in two groups: 1 . CP -12 patients with spastic CP diagnosed by clinical criteria ${ }^{5} ; 2$. Control -12 healthy patients, physical status ASA I. Exclusion criteria were systemic diseases, use of benzodiazepines or baclofen, counterindication for inhalational anesthesia, hyperthermia or obesity.

Patients were not premedicated. Anesthesia was induced and maintained with sevoflurane associated to $60 \% \mathrm{~N}_{2} \mathrm{O}$ and 3 L. min $^{-1}$ flow, valve circuit and $\mathrm{CO}_{2}$ absorber. All patients were intubated. In all cases, a regional anesthesia (simple epidural, or brachial plexus block) was associated with ropivacaine or bupivacaine in standardized doses $\left(\leq 2 \mathrm{mg} \mathrm{kg}^{-1}\right)$. Continuous monitoring consisted of end tidal $\mathrm{CO}_{2}\left(\mathrm{P}_{\mathrm{ET}} \mathrm{CO}_{2}\right)$ and sevoflurane, nasopharyngeal temperature (degrees Celsius), pulse oximetry and non-invasive blood pressure $(\mathrm{mmHg})$. Assisted ventilation was adjusted to installed to maintain $\mathrm{P}_{\mathrm{ET}} \mathrm{CO}_{2}$ between 30 and $50 \mathrm{mmHg}$.

BIS and SEP waves were evaluated in the immediate preanesthetic period whenever possible, and at $1.2 \%$ and $2.5 \%$ sevoflurane concentrations. It was administered in increments, being end tidal concentration maintained for 15 minutes before measurements. Measurements were obtained before surgery in most patients. Measurements obtained after skin incision showed no change induced by surgical stimulation due to regional anesthesia association. Baseline values, equivalent to awaken patients, were considered when patients had reactions typical of emergence, BIS was above 95, SEP waves had no depression and anesthetic alveolar concentration was 0 (zero).

Bispectral index was obtained using a A-1000 (Aspect Medical System, Natick, MA) Monitor, with referential set Zipprep leads (Aspect Medical System, Natick, MA) were fixed in Fp1, Fpz and Fp2 positions (international 10-20 system). Impedance was maintained below $2 \Omega$ and frequency bandwidth between 05 and $30 \mathrm{~Hz}$. Bispectral index from 0 to 100 and BIS two channels waves were recorded in successive times with 2 seconds of duration and updated every 5 seconds. The value considered for the study was the mean of values found in 45 seconds preceding the scheduled moment for measurement. Data were recorded in a proper form designed for the study.

Median nerve SEP was recorded with a Dantek-Keypoint release 1.6 device (Denmark), with surface leads placed in the following positions: Erb's point, CII and C3' or C4 (International $10-20$ system). Leads impedance was maintained below $5 \Omega$ and $F z$ was used as reference. Stimulation intensity was adjusted to obtain motor response threshold (Thumb Twitch). For each SEP, 300 to 500 constant current pulses were repeated with $0.2 \mathrm{~ms}$ duration and $5 \mathrm{~Hz}$ discharge ratio. Filtering bandwidth was adjusted between $20 \mathrm{~Hz}$ (low) and $3000 \mathrm{~Hz}$ (high). Signal was amplified to allow a $5 \mu \mathrm{v}$ sensitivity per division, with a screening of $5 \mathrm{~ms}$ per division. Means were calculated twice. Latency (ms) and amplitude $(\mu \mathrm{v})$ were measured for waveforms N9, N13, N19 and P/N22 at anesthetic end tidal concentrations determined in the study design. These records were obtained approximately 1 minute after BIS. Results were then printed in paper and saved in the machine's magnetic disk for afterward analysis by a neurophysiologist blind to patients' conditions.

Statistical analysis was performed with the aid of a SPSS program. Means and standard deviations of variables considered for analysis were used, except for SEP waves. Since absolute SEP values may vary with age, latency and amplitude values considered for analysis were mean percentage variation of such variables after increasing anesthetic alveolar concentration to $2.5 \%$ and maintaining it for 15 minutes, that is, mean percentage value of decreased amplitude and increased latency when anesthetic end tidal concentration was increased from $1.2 \%$ to $2.5 \%$. Simple Student's $t$ test was used for comparisons between groups and multiple measurements test was used for comparisons within groups in different sevoflurane concentrations. Multiple regression analysis and ANOVA were also used when needed.

\section{RESULTS}

There were no differences between groups in age, gender, weight and body temperature which, in all cases, was maintained above $35.3^{\circ}$ (Table I).

Table I - Patients Distribution in Both Groups - Cerebral Palsy and Control

\begin{tabular}{|c|c|c|}
\hline & CP Group & Control Group \\
\hline Gender M/F & $7 / 5$ & $4 / 8$ \\
\hline Weight (kg) * & $29.95 \pm 14.46$ & $40.91 \pm 15.95$ \\
\hline Age (years) * & $9.67 \pm 4.21$ & $12.08 \pm 3.6$ \\
\hline
\end{tabular}

* Values expressed in Mean \pm SD

CP group comprised diplegic (5), tetraplegic (4) and hemiplegic (3) patients. From those, 4 were under anticonvulsivants (phenobarbital, valproic acid and carbamazepine). 
BIS values in different concentrations proposed by the study are shown in table II. There was a mean value decrease within each group as anesthetic alveolar concentration was increased.

Mean BIS in the CP group was lower as compared to Control group, and difference was significant at sevoflurane $2.5 \%$ concentration $(p<0.05)$. Mean BIS was also lower in the CP group at $1.2 \%$ concentration, however with no statistical significance.

Table II - Comparison of Mean and Standard Deviation of Bispectral Index (BIS) in Different Anesthetic Concentrations, in Both Groups

\begin{tabular}{lcc}
\hline Sevoflurane Concentration & BIS - Cerebral Palsy & BIS - Control \\
\hline $0 \%$ & $97.75(0.45)$ & $97.58(0.67)$ \\
$1.2 \%$ & $55(13.07)$ & $60.17(9.21)$ \\
$2.5 \%$ & $30.33(7.06)$ & $37.50(9.41)^{*}$ \\
\hline
\end{tabular}

${ }^{*} p<0.05$ - significant

Baseline SEP was not possible in some patients, the reason why we decided to analyze results obtained during both sevoflurane concentrations. SEP waves behaved as expected for both groups, with increased latency and decreased amplitude when anesthetic concentration was increased (Figures 1 and 2).

Percentage amplitude variation was similar for both groups. As to SEP waves latency, those originated from thalamus-cortical derivations had a statistically significant increase in both groups when anesthetic concentration was increased (Tables III and IV). It could also be observed a trend to higher percentage increases in the $\mathrm{CP}$ group although without statistically significant differences.
Table III - Comparison of Percentage Variations $(\Delta \%)$ in Mean Amplitude Values of Different SEP Waveforms between Cerebral Palsy and Control Groups when Sevoflurane end Alveolar Concentration was Increased from $1.2 \%$ to $2.5 \%$

\begin{tabular}{lcc}
\hline SEP Waves & $\Delta \%$ Cerebral Palsy Group & $\Delta \%$ Control Group \\
\hline N9 & $-10 \%$ & $-7.9 \%$ \\
N13 & $-3.5 \%$ & $-8.8 \%$ \\
N19 & $-50.7 \%$ & $-42 \%$ \\
P/N22 & $-40.7 \%$ & $-40.7 \%$ \\
\hline
\end{tabular}

Table IV - Comparison of Percentage Variations $(\Delta \%)$ in Mean Latency Values of Different SEP Waveforms between Cerebral Palsy and Control Groups when Sevoflurane end Alveolar Concentration was Increased from $1.2 \%$ to $2.5 \%$

\begin{tabular}{lcc}
\hline SEP Waves & $\Delta \%$ Cerebral Palsy Group & $\Delta \%$ Control Group \\
\hline N9 & $4.0 \%$ & $0.1 \%$ \\
P/N13 & $3.1 \%$ & $2.8 \%$ \\
N19 & $13.2 \%$ & $9.7 \%$ \\
P/N22 & $11.9 \%$ & $10.5 \%$ \\
\hline
\end{tabular}

\section{DISCUSSION}

Our study has shown that, in the studied population, CP patients showed more pronounced electroneurophysiological changes as compared to healthy patients when submitted to the same sevoflurane end tidal concentrations. BIS decrease was more intense in the CP group, suggesting higher sensitivity of those patients to anesthetic action.
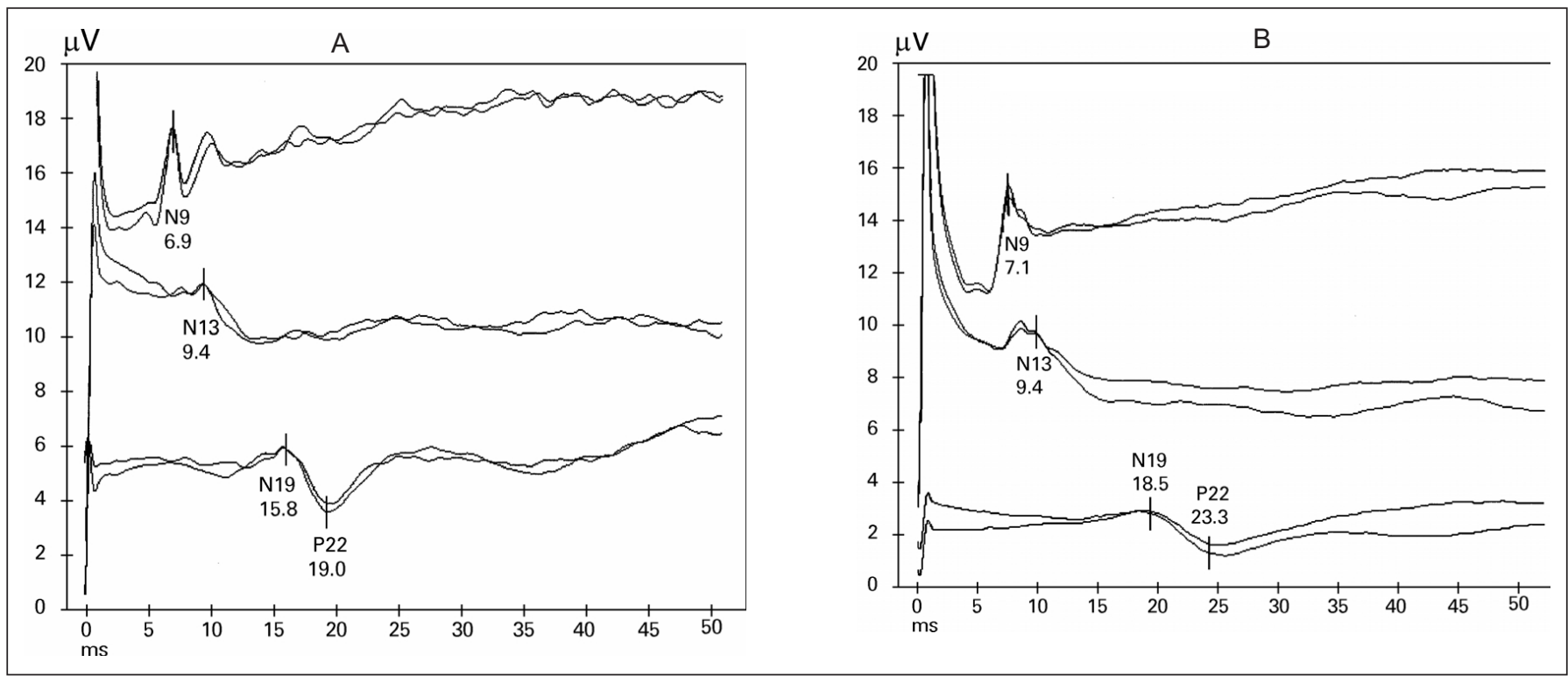

Figure 1 - SEP Waves of Control Group Patient when Awaken (A) and Anesthetized with Sevoflurane in End Tidal Concentration of 2.5\% (B), Respectively.

It is observed an increase in latency (number below wave name. Ex: N9-6,9) and decrease in amplitude in the anesthetized patient, especially in thalamus-cortical derivations (N19 and P/N22) 


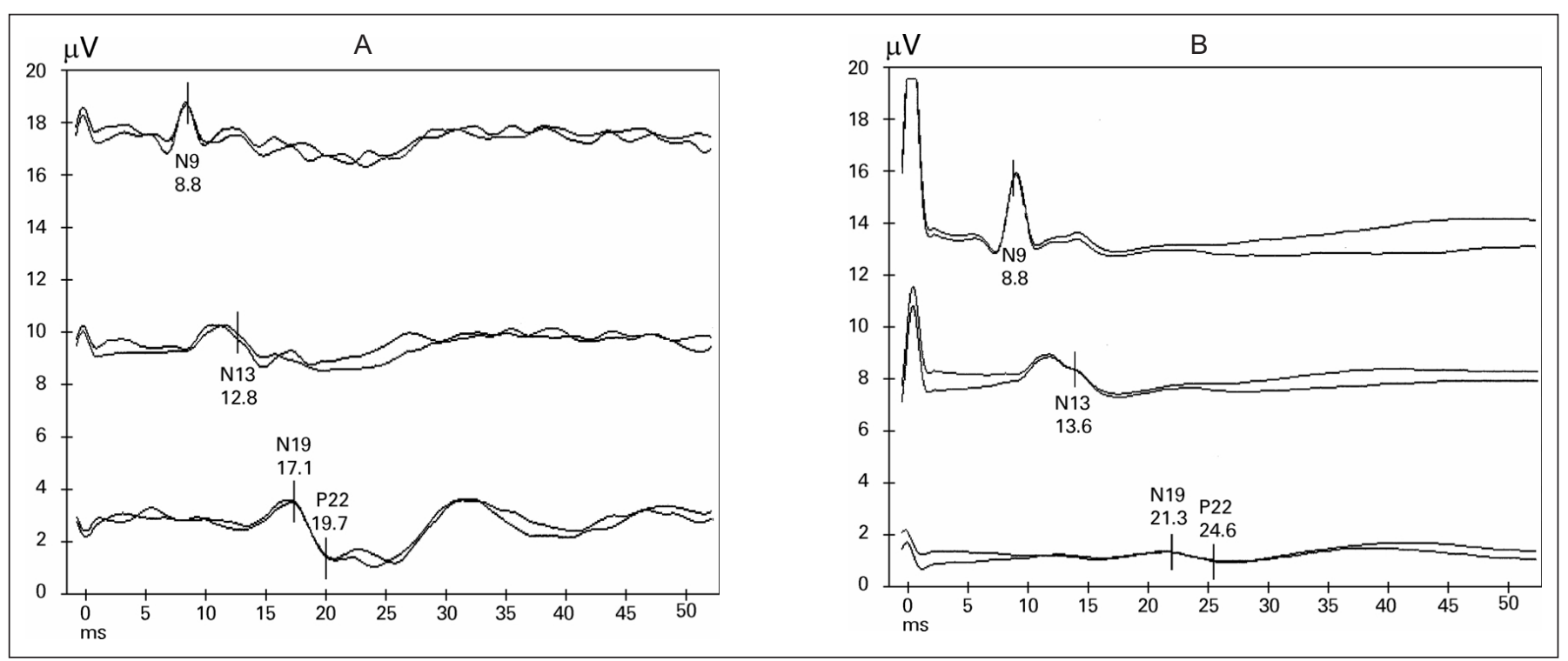

Figure 2 - SEP Waves of Cerebral Palsy Patient when Awaken (A) and Anesthetized with Sevoflurane in End Tidal Concentration of 2.5\% (B), Respectively.

It is observed an increase in latency (number below wave name. Ex: N9-6,9) and decrease in amplitude in the anesthetized patient, especially in thalamus-cortical derivations (N19 and P/N22). Such changes are more important in this group as compared to the control group

There are few studies on anesthetic drugs pharmacodynamics in CP patients. From all anesthetic drugs, neuromuscular blockers are probably those which had the larger number of published studies ${ }^{6,7}$. Also, widely used drugs in $\mathrm{CP}$, such as baclofen and benzodiazepines, were studied in terms of their interaction with inhalational agents ${ }^{8}$. Other anticonvulsivants, such as carbamazepine or valproic acid, either have not shown major effects on inhalational anesthetics when in chronic use, or had not proven their interaction with inhalational agents ${ }^{9}$. Nolan has recently publisheda review about $\mathrm{CP}$ anesthesia and calls the attention to the peculiarities involving the anesthetic management of those patients, however, without detailing anesthetic pharmacodynamic and pharmacokinetic characteristics in this specific group of patients ${ }^{8}$.

Frei et al. ${ }^{2}$, in 1997, have studied CP and severe mental retardation children in terms of sensitivity to halothane using MAC concept to evaluate the potency of the drug. However, to obtain MAC, an adequate motor response would be necessary. The use of this measurement device in CP patients, in whom major clinical manifestations are movement disorders, may bring about a major bias.

Our study tried to use techniques able to record and quantify sevoflurane-induced electroneurophysiological changes which would not depend on subjective responses or on responses which could be modified by patients' clinical conditions. So, we decided to use BIS and SEP, which record cortical and subcortical structures electric activity, respectively, and have their parameters provenly changed by inhalational agents in a dose-dependent manner, thus allowing a broader recording of CNS changes as a function of anesthetic action ${ }^{10,11}$.
We could not find any study in the literature using BIS in CP patients. In our study, this index has shown to be an adequate neurophysiological monitor for those patients. Baseline values were almost identical in both groups and there was a linear decrease as anesthetic end tidal concentration was increased, both in healthy and CP patients. In 2000, Denman et al. have validated the use of BIS in children below 2 years of age, based on the pattern of the bispectral index in those patients, which would be similar to that in older children and adults ${ }^{12}$. From our study, and using the same reasoning, we may suggest that BIS could be used in CP children, regardless to its clinical presentation, since we had in our sample patients with different CP presentations.

During anesthesia groups had different BIS patterns in terms of sensitivity to the anesthetic agent, with lower mean values the CP group as compared to Control group. This difference was found in both sevoflurane concentrations, being statistically significant only at 1 MAC though. Some hypotheses could explain such findings. Woodforth et al., in 1999, published a study showing evidences that general anesthetics with hypnotic profile, such as sevoflurane, would act by suppressing brain cortex interneuronal activity, and this would be the mechanism responsible for drug-induced unconsciousness ${ }^{13}$. Animal studies have suggested that this effect would primarily occur on cortical interneurons of laminae III and $\mathrm{V}^{14}$.

Miller, in 1998, raised the hypothesis that learning difficulties presented by CP children could be due to impairment in cortical association fibers function ${ }^{16}$. In addition, Volpe has suggested that subcapsullary neuron injuries would affect cortical organization and neuronal connectivity ${ }^{16}$. 
In comparing information from those studies, one may raise the hypothesis that CP children would already have their cortical interneuronal connections impaired. As a consequence, the amount of anesthetics needed to block those cells, previously injured and with a possible lower number of interconnections, would be lower in those patients as compared to patients in whom neuron associations are preserved. It is a promising hypothesis, but remains still to be proven.

A CNS injury in a more specific site, where general anesthetics would also act, could decrease anesthetic needs as well. Newman called the attention to the importance of the thalamus and its cortical connections for the mechanism of attention and consciousness ${ }^{17}$. Angel has also mentioned thalamus as a possible anesthetic action site in the $\mathrm{CNS}^{14}$. In some clinical CP presentations, such as spastic and choreoaterosic tetraplegia, there might be thalamus injury or dysfunction ${ }^{15}$. Changes in these structures function could justify a lower anesthetic requirement to block cells work and obtain unconsciousness recorded by BIS.

SEP data have confirmed other authors' data ${ }^{11}$. With the increase of anesthetic alveolar concentration, amplitude values have considerably decreased in both groups, as expected, and there was an increase in waveforms latency, especially in those related to thalamus-cortical generators (N12/P22). In our study, all CP group waveforms had a higher latency increase, in percentage, as compared to children without CNS disease, however without statistical significance.

Our study has limitations. There could have been prevalence bias, since only surgical CP patients were included in the sample. However, different degrees of severity and different clinical presentations of the disease were part of the study, allowing response evaluation in different disease condition. In addition, BIS is still a device being studied and improved, with limitations for its routine use in Anesthesiology ${ }^{18,19}$. Anticonvulsivants used by some $\mathrm{CP}$ patients could have changed some electrophysiological parameters. However, those under drugs knowingly decreasing MAC, such as benzodiazepines, were excluded from the study.

$\mathrm{CP}$ patients were more sensitive to sevoflurane action on CNS as compared to healthy patients when BIS and SEP were used to monitor anesthetic-induced electroneurophysiological changes. These findings may be due to a decrease in neuronal interconnections in those patients. Further studies should be carried out to clarifying this hypothesis, as long as they could not only answer this question, but also other important issues, such as those related to the action site and mechanism of inhalational anesthetics.

\section{REFERÊNCIAS - REFERENCES}

01. Bax COM - Terminology and classification of cerebral palsy. Dev Med Child Neurol, 1964; 6:295-297.

02. Frei FJ, Haemmerle MH, Brunnner R - Minimum alveolar concentration for halothane in children with cerebral palsy and several mental retardation. Anaesthesia, 1997;52:1056-1060.
03. Katoh T, Suzuki A, Ikeda K - Electroencephalographic derivates as a tool for predicting the depth of sedation and anesthesia induced by sevoflurane. Anesthesiology, 1998;88:642-650.

04. Vaughan DJA, Thornton C, Wright JR et al - Effects of different concentrations of sevoflurane and desflurane on subcortical somatosensory evoked responses in anaesthetized, non-stimulated patients. Br J Anaesth, 2001;86:59-62.

05. Campos da Paz Jr A, Burnett SW, Nomura AM - Neuromuscular Affections in Children, em: Duthie RB - Mercer's Orthopedics Surgery. $1^{\text {st }}$ Ed, London; Oxford University Press, 1996; 399-474.

06. Theroux MC, Brandom BW, Zagnoev M et al - Dose response of succinylcholine at the adductor pollicis of children with cerebral palsy during propofol and nitrous oxide anesthesia. Anesth Analg, 1994;79:761-765.

07. Dierdoff F, McNiece WL, Rao CC - Effect of succinylcholine on plasma potassium in children with cerebral palsy. Anesthesiology, 1985;62:88-90.

08. Nolan J, Chalkiadis GA, Low J et al - Anesthesia and pain management in cerebral palsy. Anaesthesia, 2000;55:32-41.

09. Viegas $O$, Stoelting RK - Halothane MAC in dogs unchanged by phentobarbital. Anesth Analg, 1976;55:677-679.

10. Bannister CF, Brosius KK, Sigl JC et al - The effect of bispectral index monitoring on anesthetic use and recovery in children anesthetized with sevoflurane in nitrous oxide. Anesth Analg, 2001;92:877-881.

11. Costa VV, Saraiva RA, Almeida AC et al - The effects of nitrous oxide on the inhibition of somatosensory evoked potential by sevoflurane in children. Anaesthesia, 2001;56:202-207.

12. Denman W, Swanson E, Rosow D et al - Pediatric evaluation of the bispectral index (BIS) monitor and correlation of BIS with end-tidal sevoflurane concentration in infants and children. Anesth Analg, 2000;90:872-877.

13. Woodforth IJ, Hicks RG, Crawford MR et al - Depression of I waves in corticospinal volleys by sevoflurane, thiopental, and propofol. Anesth Analg, 1999;89:1182-1189.

14. Angel A - Central neuronal pathways and the process of anaesthesia. Br J Anaesth, 1993;71:148-163.

15. Miller G - Cerebral Palsies an Overview, em: Miller G, Clarck GD - The Cerebral Palsies. Causes, Consequences and Management. Boston, Butterworth-Heinemann, 1998;1-35.

16. Volpe JJ - Subplate neurons: missing link in brain injury of premature infant? Pediatrics, 1996;97:112-113.

17. Newman J - Thalamic contributions to attention and consciousness. Conscious Cogn, 1995;41:72-93.

18. Chamoun NG - The position of aspect. Anesthesiology, 2000; 92:897-898.

19. Bruhn J, Bouillon T, Shafter SL - Electromyographic activity falsely elevates the bispectral index. Anesthesiology, 2000;92: 1485-1491.

\section{RESUMEN}

Mello SS, Saraiva RA - Alteraciones Eletroneurofisiológicas en Anestesia con Sevoflurano: Estudio Comparativo entre Pacientes Saludables y Pacientes con Parálisis Cerebral

Justificativa y Objetivos - Estudios evaluando la farmacodinámica de drogas anestésicas en Parálisis Cerebral $(P C)$ son escasos. El objetivo de este estudio fue comparar la respuesta eletroneurofisiológica de pacientes normales y con $P C$ a la anestesia con sevoflurano, utilizando el EEG bispectral (BIS) y potencial evocado somatosensitivo de corta latencia (PESS) como técnica de monitorización del grado de profundidad anestésica. 
Método - Fueron seleccionados 24 pacientes de 3 a 18 años, candidatos a cirugías en la Red Sarah de Hospitales del Aparato Locomotor, divididos en 2 grupos: 1. PC - 12 niños con PC tipo espástica; 2. Control (C) - 12 niños sin enfermedad del SNC. La anestesia fue realizada con sevoflurano asociado a $\mathrm{N}_{2} \mathrm{O}$ a $60 \%$ en ventilación asistida. Las variables BIS y ondas N9, N13, N19 y P/N 22 del PESS - latencia y amplitud - fueron evaluadas en las fracciones expiradas de sevoflurano (FEsev) de 1,2 y $2,5 \%(0,5$ y 1 CAM). Fueron monitoradas la temperatura y la $P_{E T} C_{2}$. Para análisis fueron utilizadas media y desvio-patrón para el BIS, y media de la variación porcentual de los valores de latencia y amplitud de las ondas del PESS, en las dos concentraciones del anestésico.

Resultados - No hubo diferencia de sexo, edad, peso y temperatura entre los grupos. Bajo efecto de la anestesia, los valores de BIS fueron mas reducidos en el grupo PC, siendo la diferencia entre los grupos estadísticamente significante con la FEsev 2,5\% (30,3 $\times 37,5 ; p<0,05)$. El aumento porcentual de la latencia de las ondas del PESS fue mayor en el grupo PC.

Conclusiones - Los resultados sugieren un efecto depresor mayor del anestésico en el grupo con PC, siendo esta diferencia captada pelo BIS y PESS. 